OPEN ACCESS

Edited by:

Nathalie Connil,

Université de Rouen, France

Reviewed by

Christopher Gaulke,

University of Illinois

at Urbana-Champaign, United States

Huan Li,

Lanzhou University, China

*Correspondence:

Luigimaria Borruso

luigimaria.borruso@unibz.it

Paola Mattarelli

paola.mattarelli@unibo.it

Specialty section: This article was submitted to

Systems Microbiology,

a section of the journal

Frontiers in Microbiology

Received: 15 February 2021

Accepted: 08 July 2021

Published: 06 August 2021

Citation:

Correa F, Torti V, Spiezio C, Checcucci A, Modesto M, Borruso L,

Cavani L, Mimmo T, Cesco S,

Luise D, Randrianarison RM,

Gamba M, Rarojoson NJ,

Sanguinetti $M$, Di Vito $M$, Bugli F, Mattarelli $P$, Trevisi $P$, Giacoma $C$ and

Sandri C (2021) Disentangling

the Possible Drivers of Indri indri Microbiome: A Threatened Lemur

Species of Madagascar.

Front. Microbiol. 12:668274. doi: 10.3389/fmicb.2021.668274

\section{Disentangling the Possible Drivers of Indri indri Microbiome: A Threatened Lemur Species of Madagascar}

\author{
Federico Correa1, Valeria Torti ${ }^{2}$, Caterina Spiezio ${ }^{3}$, Alice Checcucci ${ }^{1}$, Monica Modesto ${ }^{1}$, \\ Luigimaria Borruso ${ }^{*}$, Luciano Cavani ${ }^{1}$, Tanja Mimmo ${ }^{4}$, Stefano Cesco ${ }^{4}$, Diana Luise ${ }^{1}$, \\ Rose M. Randrianarison ${ }^{5,6}$, Marco Gamba $^{2}$, Nianja J. Rarojoson7, \\ Maurizio Sanguinetti, ${ }^{8,9}$, Maura Di Vito ${ }^{8}$, Francesca Bugli ${ }^{8,9}$, Paola Mattarelli ${ }^{*}$, \\ Paolo Trevisi ${ }^{1}$, Cristina Giacoma ${ }^{2}$ and Camillo Sandri ${ }^{1,3}$ \\ ${ }^{1}$ Department of Agricultural and Food Sciences, University of Bologna, Bologna, Italy, ${ }^{2}$ Department of Life Sciences \\ and Systems Biology, University of Torino, Turin, Italy, ${ }^{3}$ Department of Animal Health Care and Management, Parco Natura \\ Viva - Garda Zoological Park, Verona, Italy, ${ }^{4}$ Faculty of Science and Technology, Free University of Bolzano-Bozen, Bolzano, \\ Italy, ${ }^{5}$ Groupe d'Étude et de Recherche sur les Primates de Madagascar, Antananarivo, Madagascar, ${ }^{6}$ Mention \\ d'Anthropobiologie et de Deiveloppement Durable, Université de Antananarivo, Antananarivo, Madagascar, ${ }^{7}$ Laboratoire \\ de Pédologie, FOFIFA à Tsimbazaza, Antananarivo, Madagascar, ${ }^{8}$ Dipartimento di Scienze Biotecnologiche di Base, Cliniche \\ Intensivologiche e Perioperatorie, Università Cattolica del Sacro Cuore, Rome, Italy, ${ }^{9}$ Dipartimento di Scienze di Laboratorio \\ e Infettivologiche, Fondazione Policlinico Universitario A. Gemelli IRCCS, Rome, Italy
}

Research on the gut microbiome may help with increasing our understanding of primate health with species' ecology, evolution, and behavior. In particular, microbiome-related information has the potential to clarify ecology issues, providing knowledge in support of wild primates conservation and their associated habitats. Indri (Indri indri) is the largest extant living lemur of Madagascar. This species is classified as "critically endangered" by the IUCN Red List of Threatened Species, representing one of the world's 25 most endangered primates. Indris diet is mainly folivorous, but these primates frequently and voluntarily engage in geophagy. Indris have never been successfully bred under human care, suggesting that some behavioral and/or ecological factors are still not considered from the ex situ conservation protocols. Here, we explored gut microbiome composition of 18 indris belonging to 5 different family groups. The most represented phyla were Proteobacteria $40.1 \pm 9.5 \%$, Bacteroidetes $28.7 \pm 2.8 \%$, Synergistetes $16.7 \pm 4.5 \%$, and Firmicutes $11.1 \pm 1.9 \%$. Further, our results revealed that bacterial alpha and beta diversity were influenced by indri family group and sex. In addition, we investigated the chemical composition of geophagic soil to explore the possible ecological value of soil as a nutrient supply. The quite acidic $\mathrm{pH}$ and high levels of secondary oxide-hydroxides of the soils could play a role in the folivorous diet's gut detoxification activity. In addition, the high contents of iron and manganese found the soils could act as micronutrients in the indris' diet. Nevertheless, the concentration of a few elements (i.e., calcium, sulfur, boron, nickel, sodium, and chromium) was higher in non-geophagic than in geophagic soils. In conclusion, the data presented herein provide a baseline for outlining some possible drivers responsible for the gut microbiome diversity in indris, thus laying the foundations for developing further strategies involved in indris' conservation.

Keywords: gut microbiome, soil quality, non-human primate, animal ecology, endangered species, geophagy, forest ecology 


\section{INTRODUCTION}

Studies on human and animal microbiome have provided compelling evidence that gut microbial diversity is fundamental in shaping metabolic and regulatory networks involved in the maintenance of host healthy status, as well as in a spectrum of disease states (Shreiner et al., 2015; Sandri et al., 2020). Indeed, the mammalian gut microbiome plays a crucial role in host physiology, supporting vitamin synthesis, helping in complex carbohydrates digestion, toxins metabolism, pathogens antagonism, and immune system modulation (Cresci and Bawden, 2015). Factors influencing the differences in mammalian gut microbiome are debated: host behaviors and environments, biogeography, and host genetic effects (e.g., gastrointestinal tract morphology) are of great importance (Lankau et al., 2012; Moeller et al., 2013; Amato et al., 2016). Previous studies showed that frequent social networks are positively associated with high similarity in gut microbial diversity (Tung et al., 2015; Perofsky et al., 2019). Vertical transmission from parent to offspring is the first driver for gut microbiome development, but horizontal transmission from the environment provides a crucial microbial colonization route. Even if microbial transmission due to sociality has traditionally been viewed as a risk for pathogen exposure, it may also be essential to host health. Therefore, it can avoid bottleneck-induced extinctions that could occur when the transmission of microorganisms is strict from parent to offspring. Indeed, it can allow the acquisition of beneficial microbes, particularly those that might not be gained through vertical transmission (Lombardo, 2008; Amaral et al., 2017). Moeller et al. (2013) underlined that gut microbial populations' social inheritance might be fundamental for preserving microbial diversity over evolutionary time scales.

The lemurs harbored species-specific and/or populations specific microbiomes, which are mainly influenced by their dietary specificity, even on a seasonal basis (Fogel, 2015; Greene et al., 2020). Globally, host habitat is one of the most important factors for gut microbiome modulation, and recently, increasing attention has been devoted to the soil. Indeed, a recent study (Grieneisen et al., 2019) on the gut microbiome of terrestrially living baboons showed that bare soil exceeds 15 times the predictive ability of host genetics in shaping the gut microbiome. Studies in mice (Li et al., 2016; Zhou et al., 2018) confirmed that the effect of soil on gut microbiome composition is comparable to that exerted by diet. Therefore, these studies suggest that contact/ingestion of soil components is beneficial for a healthy gut microbiome.

Indri indri is the largest extant living lemur (Figure $\mathbf{1}$ and Supplementary Video 1). It is mainly arboreal and is the only lemur that communicates using songs. Indris songs mediate both intra- and inter-group communication (Torti et al., 2013) and relay information regarding individual features (i.e., sex and age) (De Gregorio et al., 2019, 2021). This species has never successfully been kept in a controlled environment and it is considered one of the Malagasy most critically endangered lemurs according to the IUCN Red List of Threatened Species (King et al., 2020), representing one of the world's 25 most endangered primates (Torti et al., 2019). This species is also listed in Appendix I of CITES (Heinen and Mehta, 1999). Indris are territorial, socially primates living in small family groups (Pollock, 1979; Bonadonna et al., 2019), generally consisting of an adult male and female with their related offspring (2-6 individuals) (Torti et al., 2013; Gamba et al., 2016).

Non-human primates are characterized by many dietary specializations (Campbell, 2017). In particular, the ability to consume leaves is typical of new world monkeys (e.g., howler monkeys), old world monkeys (e.g., colobines), apes (e.g., gorillas), and also prosimians (e.g., indris, bamboo lemurs, and sportive lemurs). Indri is the most specialized folivorous among lemurs and, as such, has the highest degree of morphological specialization for leaves' consumption and digestion. Leaves contain carbohydrates, including cellulose and hemicellulose, and secondary metabolites, including toxic ones such as tannins and phenolics (Norconk et al., 2009). Indris are characterized by the typical morphology and anatomical specializations of folivorous primates, such as hypertrophic salivary glands, voluminous stomachs, sacculated caeca, and looped colons that facilitate efficient fermentation of leaf matter (Greene et al., 2020). The species shows a preference for immature leaves (72\%) with a reduced emphasis on fruit seeds/whole fruits (16\%) and flowers (7\%) (Powzyk, 1997). Leaves and fruit seeds could contain toxic compounds varying in percentage depending on the season, maturity, etc. (Pebsworth et al., 2019). In addition, indris perform geophagy by consuming soil intentionally (Britt et al., 2002; Borruso et al., 2021). Some evidence suggests that geophagy is an adaptive behavior to protect from ingested toxic compounds and mineral supplementation as it facilitates consumption of plants binding toxic plant secondary compounds (PSCs) (Pebsworth et al., 2019). As a result of metabolic activity, plants with relevant antioxidant properties produce primary and secondary compounds. Nevertheless, several metabolites are universally distributed in many plant species; some are unique to individual plant cultivars and fill essential functions (Geilfus, 2019).

Studies regarding geophagy across non-human primates revealed that they eat items high in PSCs. Furthermore, they consume soil more often than sympatric populations, suggesting a decrease in gastrointestinal distress caused by PSCs. Geophagy can help the utilization of dietary resources high in PSCs, expanding the range of dietary components (Overdorff, 1993; Bocian, 1997; Powzyk and Mowry, 2003; Dew, 2005; Pebsworth et al., 2019). In addition to dietary toxins, mineral deficiencies, diarrhea, and altered gut $\mathrm{pH}$ were reported to cause geophagy (Krishnamani and Mahaney, 2000; Ferrari et al., 2008; Young et al., 2011). As these processes are not necessarily mutually exclusive, geophagy can play different functions, such as rare element supplementation, detoxification, and protection (Davies and Baillie, 1988; Huffman et al., 1997; Krishnamani and Mahaney, 2000; Pebsworth et al., 2019). Interestingly, geophagic soil could also be a reservoir for microbial species affecting indris' gut microbiome (Borruso et al., 2021). The highly specialized diet, physiology, and morphology of indri's gut may contribute to their susceptibility in a human-controlled environment. This is in analogy for what has been described for other endangered folivorous primate whose breeding was unsuccessful (Hale et al., 2018, 2019). 


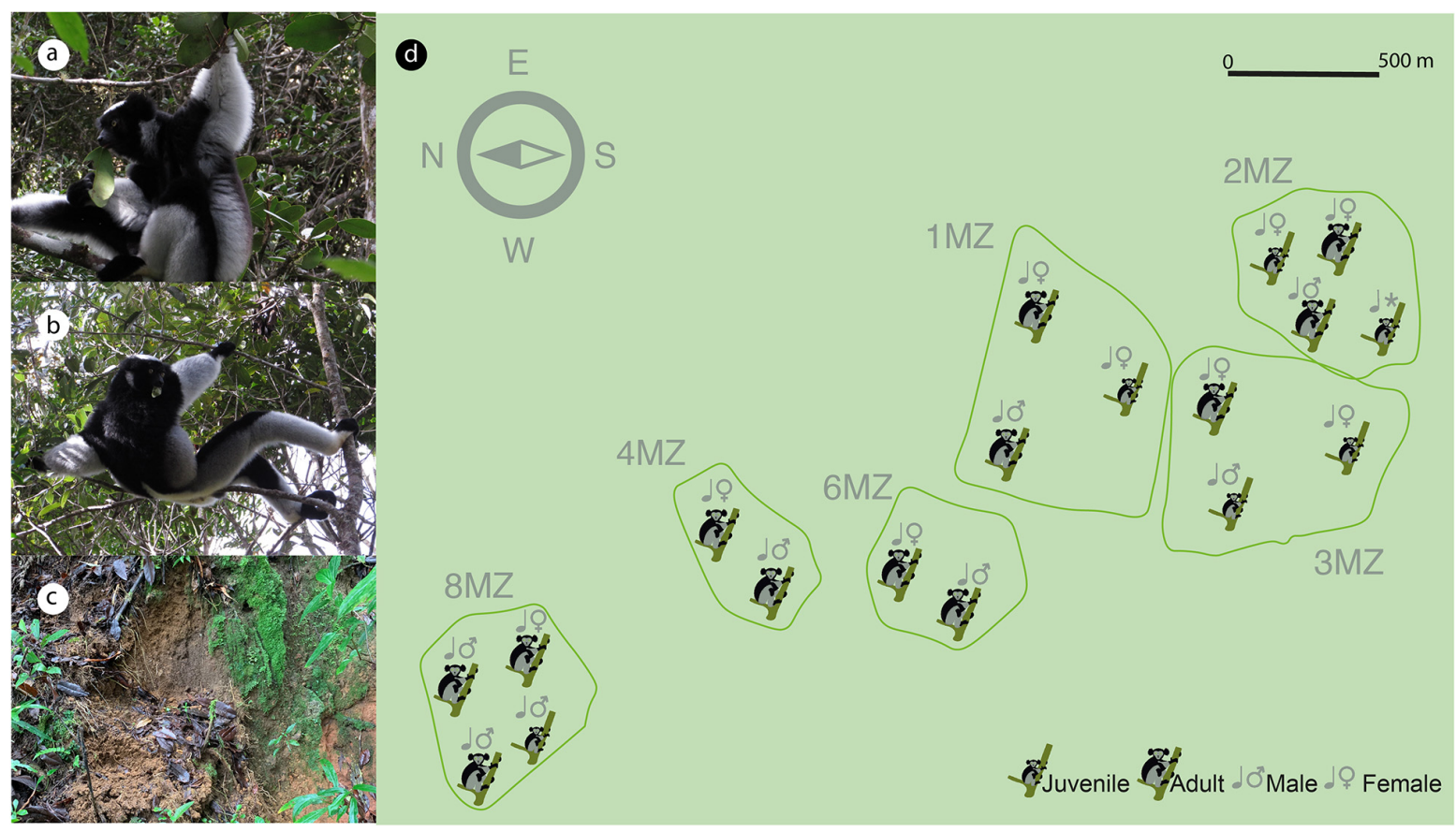

FIGURE 1 | Distribution of the territories occupied by the indri family groups sampled (main figure, d) and composition of each single group. Both adult and youngster indris, both sexes, feed on leaves $\mathbf{( a , b )}$ and perform geophagic behavior, eating soil in specific sites (c).

Understanding the drivers of the gut indris microbiome and their relationship to the soil could be essential for planning strategies to conserve, monitor, and promote their health. Whether the gut microbiome facilitates the use of these hardto-digest food items, it would be crucial to characterize the bacterial gut microbiome's shaping factors. Therefore, our work aimed to analyze: (1) the gut microbiome composition of wild indris belonging to five different familiar groups in Maromizaha, eastern Madagascar; (2) the potential drivers affecting hostmicrobial diversity, including sex, family group, and age class (3) the chemical composition of geophagic and non-geophagic soil, to unravel the possible adaptive ecological value as nutrient supply.

\section{MATERIALS AND METHODS}

\section{Fecal and Soil Samples Collection}

Fecal and soil samples were collected in a very narrow temporal window (between December 4th and 6th, 2018) to avoid confounding potential seasonal effects. Individual fecal samples were obtained from 18 indris (fecal material) belonging to 5 different social family groups (Table $\mathbf{1}$ and Figure 1) (latitude $18^{\circ} 57^{\prime} \mathrm{S}$ and $19^{\circ} 00^{\prime} \mathrm{S}$, longitude $48^{\circ} 26^{\prime} \mathrm{E}$ and $48^{\circ} 31^{\prime} \mathrm{E}$, Madagascar). The samples were collected immediately after defecation, when only one animal, recognized using natural marks (Torti et al., 2013), was present. This procedure was essential to avoid individual misidentification during the sampling process (Bonadonna et al., 2019). Approximately $5 \mathrm{~g}$ of fecal samples were collected from each of the 18 individuals
(Table 1) following the procedure described in Borruso et al. (2021).

Each sample was classified according to the following categories: sex, family group, and age class (Adult $>6$ years and Juvenile $<6$ years) (Table 1 and Figure 1). In addition, soil samples were collected from seven geophagic and seven nongeophagic (control) sites. All the geophagic sites were at the bases of trees uprooted by wind or rainfall, with the lower soil horizons exposed. We noted the location (waypoint) during soileating events, and we followed behaviors before and after the geophagy event. Control sites were selected from areas with the same characteristics (slope, vegetation, etc.) and located at less than $20 \mathrm{~m}$ from geophagic sites after removing the superficial soil layer to sample the same soil layer of the geophagic sites. The presence of the superficial layer together with debris proved that the groups have never used the control locations to consume soil. All samples were maintained in a portable cooler with ice packs before arrival at the lab.

\section{Soil Characterization}

Soil samples were air-dried, milled, and sieved at $2 \mathrm{~mm}$ for soil analysis in agreement with Soil Science Society of America (SSSA) methods (Sparks et al., 1996). Briefly, pH was determined in water $(1: 2.5, \mathrm{~m} / \mathrm{V})$, total carbon $(\mathrm{C})$, and total nitrogen (N) using an elemental analyzer (CHNS-O Elemental Analyzer 1110, Thermo Scientific GmbH, Germany). Pseudo total element concentrations were determined after acid mineralization with aqua regia and hydrogen peroxide in an Ethos TC microwave lab station (Milestone, Italy) using an inductively coupled plasma 
TABLE 1 | Description of each indri individual including sex, class age (Adult, > 6 years; Juvenile, $<6$ years; NA*, not available) and family group, bacterial observed richness, and bacterial Shannon index values.

\begin{tabular}{|c|c|c|c|c|c|}
\hline Samples ID & Sex & Class age & Family group & Observed richness & Shannon \\
\hline $\mathrm{L}$ & Female & Adult & $1 \mathrm{MZ}$ & 44 & 2.87 \\
\hline$M$ & Female & Juvenile & $1 \mathrm{MZ}$ & 41 & 2.55 \\
\hline $\mathrm{O} 2$ & Male & Adult & $1 \mathrm{MZ}$ & 43 & 2.11 \\
\hline $\mathrm{R}$ & Female & Juvenile & $2 \mathrm{MZ}$ & 44 & 2.55 \\
\hline N2 & $N A^{*}$ & Juvenile & $2 \mathrm{MZ}$ & 44 & 2.76 \\
\hline$P$ & Male & Adult & $2 \mathrm{MZ}$ & 46 & 2.67 \\
\hline Q & Female & Adult & $2 \mathrm{MZ}$ & 47 & 2.90 \\
\hline $\mathrm{G}$ & Female & Juvenile & $3 \mathrm{MZ}$ & 35 & 2.55 \\
\hline $\mathrm{H}$ & Male & Juvenile & $3 \mathrm{MZ}$ & 37 & 1.95 \\
\hline I & Female & Adult & $3 \mathrm{MZ}$ & 35 & 2.41 \\
\hline C & Female & Adult & $4 \mathrm{MZ}$ & 38 & 2.70 \\
\hline E2 & Male & Adult & $4 \mathrm{MZ}$ & 45 & 2.40 \\
\hline$K$ & Female & Adult & $6 \mathrm{MZ}$ & 47 & 2.75 \\
\hline S & Male & Adult & $6 \mathrm{MZ}$ & 39 & 2.59 \\
\hline $\mathrm{A} 2$ & Female & Adult & $8 M Z$ & 55 & 2.94 \\
\hline B2 & Male & Juvenile & $8 M Z$ & 58 & 2.69 \\
\hline D2 & Male & Adult & $8 \mathrm{MZ}$ & 55 & 2.62 \\
\hline $\mathrm{F} 2$ & Male & Adult & $8 M Z$ & 58 & 2.89 \\
\hline
\end{tabular}

optical emission spectrometer (ICP-OES, Ametek Spectro, Arcos, Germany). Available metals were determined by ICP-OES after extraction for $2 \mathrm{~h}$ with $1 \mathrm{~mol} \mathrm{~L}-1$ ammonium nitrate $\left(\mathrm{NH}_{4} \mathrm{NO}_{3}\right)$ solution $(1: 2.5, \mathrm{~m} / \mathrm{V})$.

\section{DNA Extraction and NGS Sequencing}

Total DNA was isolated and extracted from indri fecal samples with DNeasy PowerSoil Kit (QIAGEN, Hilden, Germany) with slight modifications. Briefly, the lysis step was enhanced using a bead-beater (FastPrep 24G, MP Biomedicals, France), in which the "Powerbead" tubes containing the pellets $(250 \mathrm{mg}$ of fecal sample) and $800 \mu \mathrm{L}$ of CD1 solution were subjected to two cycles of bead-beating at a speed of $4 \mathrm{~m} / \mathrm{s}$ for $60 \mathrm{~s}$ with $45 \mathrm{~s}$ pause between cycles. The final elution volume was $100 \mu \mathrm{L}$ in water. DNA was checked for purity (absorbance ratio 260/280 and 260/230) by spectrophotometry using NanoDrop (Fisher Scientific, 13 Schwerte, Germany) and quantified with the fluorometer Qubit ${ }^{\circledR} 2.0$ (Invitrogen, Italy). Next, the DNA concentration of each sample was normalized to $1 \mathrm{ng} \mu \mathrm{L}^{-1}$. The PCR was performed amplifying the V3-V4 region of the $16 \mathrm{~S}$ rRNA gene ( $460 \mathrm{bp})$ with the primers Pro341F $\left(5^{\prime}\right.$ TCGTCGGCAGCGTCAGATGTGTATAAGAGACAGCCTAC GGGNBGCASCAG-3') and Pro805R (5'-GTCTCGTGGGCTCG GAGATGTGTATAAGAGACAGGACTACNVGGGTATCTAAT CC-3') (Takahashi et al., 2014), using Platinum Taq DNA Polymerase High Fidelity (Thermo Fisher Scientific, Italy). The thermal cycling protocol consisted of the following conditions: initial denaturation at $94^{\circ} \mathrm{C}$ for $1^{\prime}$, followed by 25 cycles of denaturation at $94^{\circ} \mathrm{C}$ for $30^{\prime \prime}$, annealing at $55^{\circ} \mathrm{C}$ for $30^{\prime \prime}$, and extension $65^{\circ} \mathrm{C}$ for $45^{\prime \prime}$, ending with 1 cycle at $68^{\circ} \mathrm{C}$ for $7^{\prime}$. Further, PCR samples were sent to BMR-Genomics Ltd., that according to the standard protocols carried out the other steps of the workflow and finally sequenced the libraries using a MiSq platform (300 × 2 bp) (Illumina Inc., San Diego, CA, United States).

The raw reads obtained are publicly available at the Sequence Read Archive (SRA) under the accession number: PRJNA701813.

\section{Bioinformatic Analysis}

Sequencing data analysis was performed using DADA2 1.14.0 (Callahan et al., 2016) running on $\mathrm{R}$ 3.6.2 (R Core Team, 2021). The forward and reverse reads were trimmed to remove low-quality nucleotides and primers sequences using the filterAndTrim function with the following parameters: truncLen $=c(290,220)$, trimLeft $=c(50,55)$, and $\operatorname{maxN}=0$, truncQ $=2$. The amplicon sequence variants were inferred using the DADA2 core sample inference algorithm with default parameters. Forward and reverse reads were merged and reads with mismatches were removed. Chimeras were identified using the removeBimeraDenovo function and removed. Further, the SILVA database release 132 (Quast et al., 2013) was used for the taxonomic assignment. Finally, the AVSs table was rarefied to 25,181 reads per sample.

\section{Statistical Analysis}

Statistical analyses were carried out using Phyloseq 1.32 (McMurdie and Holmes, 2013) and Vegan 2.5 (Dixon, 2003) packages. The differences between the geophagic and nongeophagic control soil composition were tested via MannWhitney $U$-test. Alpha diversity was explored considering the Shannon index and Observed richness calculated from the rarefied AVSs table (25,181 reads). Both indices values were checked for normality using the Shapiro-Wilk test. The possible effects of sex, age class, and family group on alpha diversity indices were evaluated with a Linear Model (ANOVA type III). Beta dispersion was calculated to test if the groups, classified 

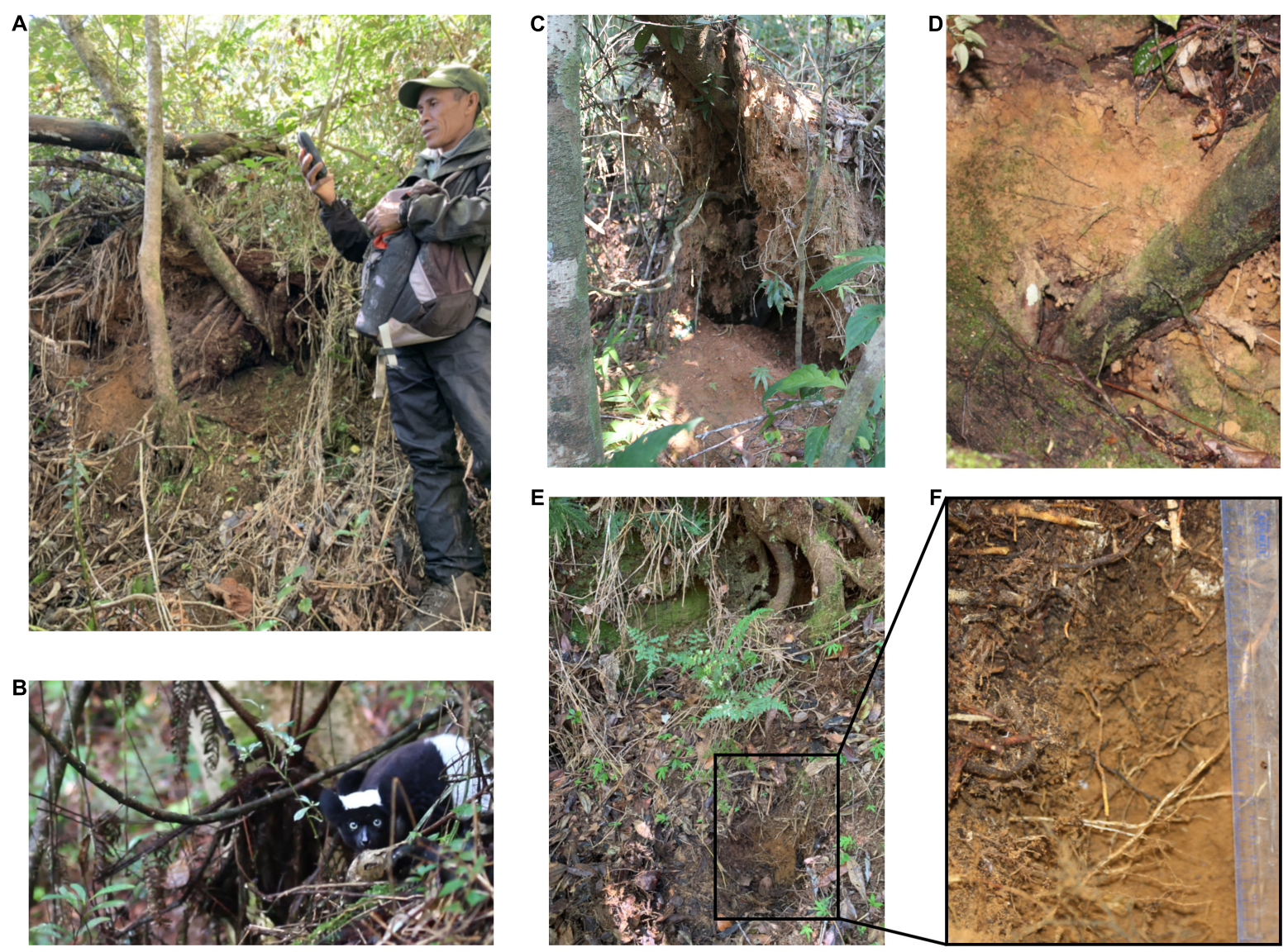

FIGURE 2 | Visualization of (A) a research guide recording the location of a geophagic site (GPS waypoint); (B) an indri (Indri indri) performing geophagic behavior, eating soil in a specific site; (C) a geophagic site under a fallen tree; (D) soil horizon eaten in the geophagic site; (E) a control site with the upper surface untouched; and $\mathbf{( F )}$ enlargement of the soil sampled in the control site, under the surface, in the horizon normally eaten by indri. Soil is collected free of debris (grass, leaves, stones, roots).

according to sex, age class, and family group, had the same centroids and heterogeneity. Permutational multivariate analysis of variance (PERMANOVA) was applied to test the possible effect of sex, age class, and family group on the bacterial communities. In addition, the Constrained Analysis of Principal Coordinates (CAP) based on Bray Curtis was used to generate the ordination plots.

Linear discriminant analysis effect size (LEfSe) algorithm (LDA score $\geq 2$ and $p$-value $<0.05$ ) was applied to detect the biomarker taxa for each category (Segata et al., 2011). We excluded from the LEfSe analysis the family groups with less than three individuals (i.e., $4 \mathrm{MZ}$ and $6 \mathrm{MZ}$ ).

\section{RESULTS}

\section{Geophagy Site Characterization}

Indris were observed to eat soil in sites at the bases of trees uprooted by wind and/or by rainfall, with the lower soil horizons exposed (Figure 2 and Supplementary Video 1). Geophagic and non-geophagic soil samples were characterized by an acidic $\mathrm{pH}$ and rich content in total $\mathrm{C}$ and $\mathrm{N}$. With regards to the pseudototal metals, soil samples showed poor content in Calcium (Ca), Phosphorus (P), Sulfur (S), and higher content in Iron (Fe). Manganese ( $\mathrm{Mn})$ and Fe were the most extractable in ammonium nitrate in the case of available metals (Supplementary Table 1).

Some differences were found between geophagic and nongeophagic sites. Specifically, the concentration of $\mathrm{Ca}$, $\mathrm{S}$, sodium $(\mathrm{Na})$, chromium $(\mathrm{Cr})$, boron $(\mathrm{B})$, and available Nickel $(\mathrm{Ni})$ resulted in being higher in non-geophagic than in geophagic soil samples ( $p$-value $<0.01$ ) (Figure 3$)$. On the other hand, for all the other parameters, including $\mathrm{pH}$, total $\mathrm{C}$, total $\mathrm{N}$, the remaining pseudo-total elements, and metals extractable in ammonium nitrate, no statistically significant differences were observed (Supplementary Table 1).

\section{Bacterial Taxonomic Community Composition}

After quality checking and filtering, 645,297 reads (including non-bacterial reads) were generated from the MiSeq run. The reads assigned as Bacteria were 616,269 resulting in 131 amplicon sequence variants (Supplementary Tables 2, 3). 

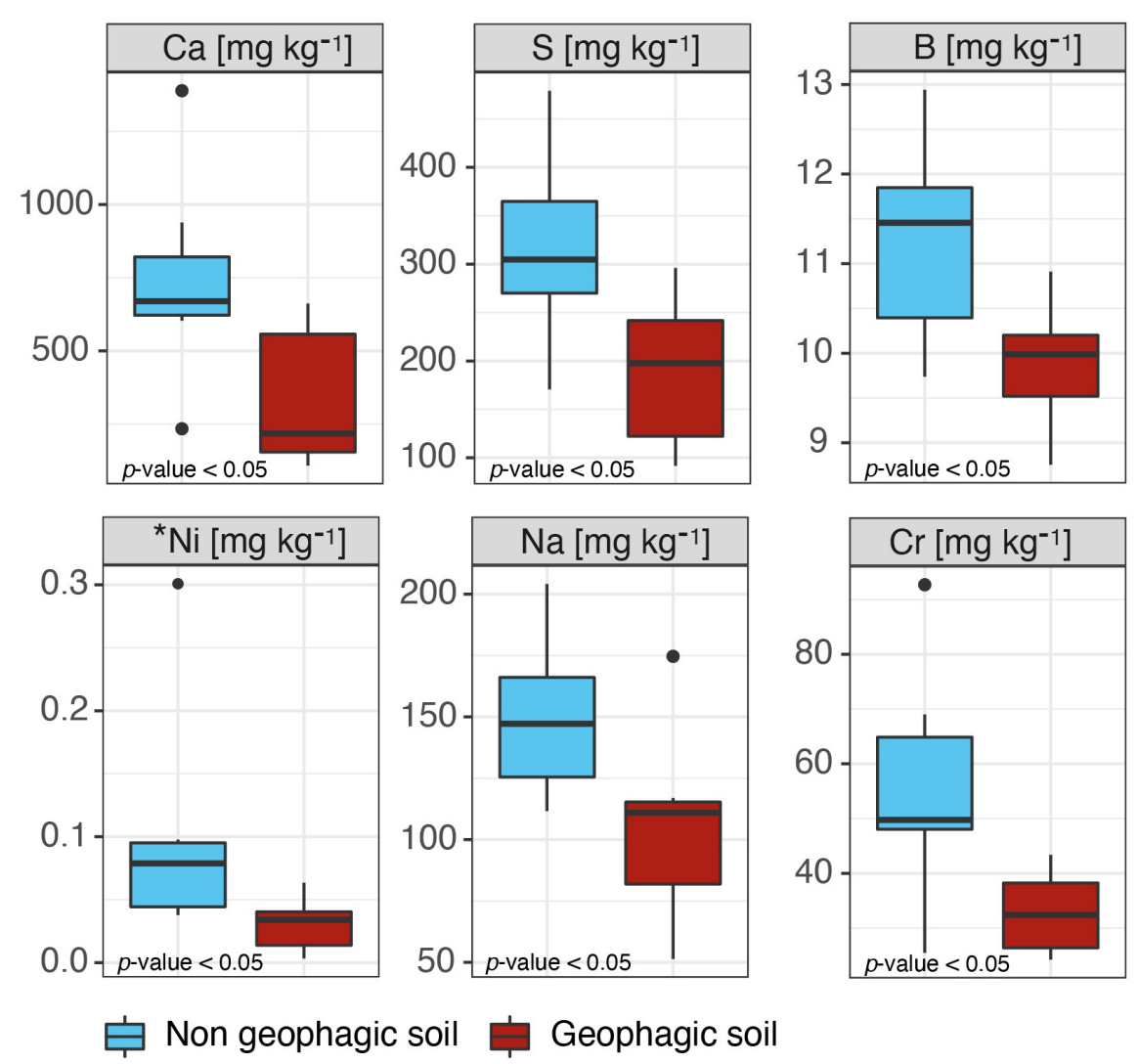

FIGURE 3 | Boxplots representing the chemical parameters resulted statistically different $(p$-value $<0.05)$ between the geophagic and non-geophagic soils. ${ }^{*}$ Available metal.

Rarefaction curves showed that all the samples nearly reached the plateau (Supplementary Figure 1). All the samples were identified at phylum level: Proteobacteria $40.1 \pm 9.5 \%$, Bacteroidetes $28.7 \pm 2.8 \%$, Synergistetes $16.7 \pm 4.5 \%$, Firmicutes $11.1 \pm 1.9 \%$, Verrucomicrobia $2.0 \pm 1.2 \%$, Actinobacteria $1.2 \pm 0.6 \%$, and Cyanobacteria $0.2 \pm 0.3 \%$ (Figure 4A). At family level the most abundant groups were: Succinivibrionaceae $39.6 \pm 11.6 \%$, Prevotellaceae $26.4 \pm 3.2 \%$, Synergistaceae $16.7 \pm 4.5 \%$, Ruminococcaceae $6.6 \pm 2.7 \%$, Acidaminococcaceae $3.3 \pm 1.2 \%$, and Puniceicoccaceae $2.0 \pm 1.2 \%$ (Figure 4B). At a finer taxonomic level, the prevalent genera identified were: Anaerobiospirillum $39.3 \pm 11.9 \%$ and Prevotellaceae NK3B31 group $19.8 \pm 3.8 \%$, Cloacibacillus $8.2 \% \pm 7.2 \%$, Ruminococcus 1, $5.0 \pm 2.8 \%$, Jonquetella $4.24 \% \pm 2.8 \%$, Pyramidobacter $4.0 \pm 2.8 \%$, Phascolarctobacterium $2.6 \pm 1.2 \%$, and Cerasicoccus $2.0 \pm 1.2 \%$ (Figure 4C).

\section{Effect of Family Group, Sex, and Age Class on Indri Bacterial Diversity}

Considering all the individuals, the mean Shannon diversity was $2.61 \pm 0.26$, whereas the Observed richness's value was $45 \pm 7$. The values for each individual are reported in Table 1. Shannon diversity and Observed richness data resulted to be normally distributed (Shapiro-Wilk normality test: Observed richness, $W=0.92, p$-value $=0.14$; Shannon diversity, $W=0.91$, $p$-value $=0.07)$.

The Linear Model revealed that Observed richness was influenced by family group $(F=17.69$, $p$-value $=0.0002)$, whereas Shannon diversity was affected by both family group $(F=4.37$, $p$-value $=0.02)$ and $\operatorname{sex}(F=10.02, p$-value $=0.01)$. In particular, females showed higher alpha diversity values if compared to males. Finally, no significant effect was detected according to the age class (Supplementary Table 4).

Beta-dispersion of bacterial communities revealed that the samples had homogeneous dispersion (Sex, $F=1.24$ and $p$-value $=0.31$; family group, $F=1.21$ and $p$-value $=0.43$; age class $F=0.002$ and $p$-value $=0.98)$. PERMANOVA analysis showed that sex $(F=7.43, p$-value $=0.001)$ and family group $(F=7.4707, p$-value $=0.001)$ resulted to significantly affect the bacterial communities's beta-diversity, differently from age class $(F=0.89, p$-value $=0.51)$. Further, CAP analysis, confirming the results obtained with the PERMANONVA, found that among all the tested possible drivers, sex, and family group influenced the bacterial community's structure (com $\sim$ family group + Sex; $F=5.94 p$-value $=0.001)($ Figure 5) .

Linear discriminant analysis effect size algorithm found 15 ASVs biomarkers for the group 1MZ, 17 ASVs with 2MZ, 11 ASVs with 3MZ, and 25 with $8 \mathrm{MZ}$ (Supplementary Table 5). At phylotype level, Proteobacteria, mainly with the 


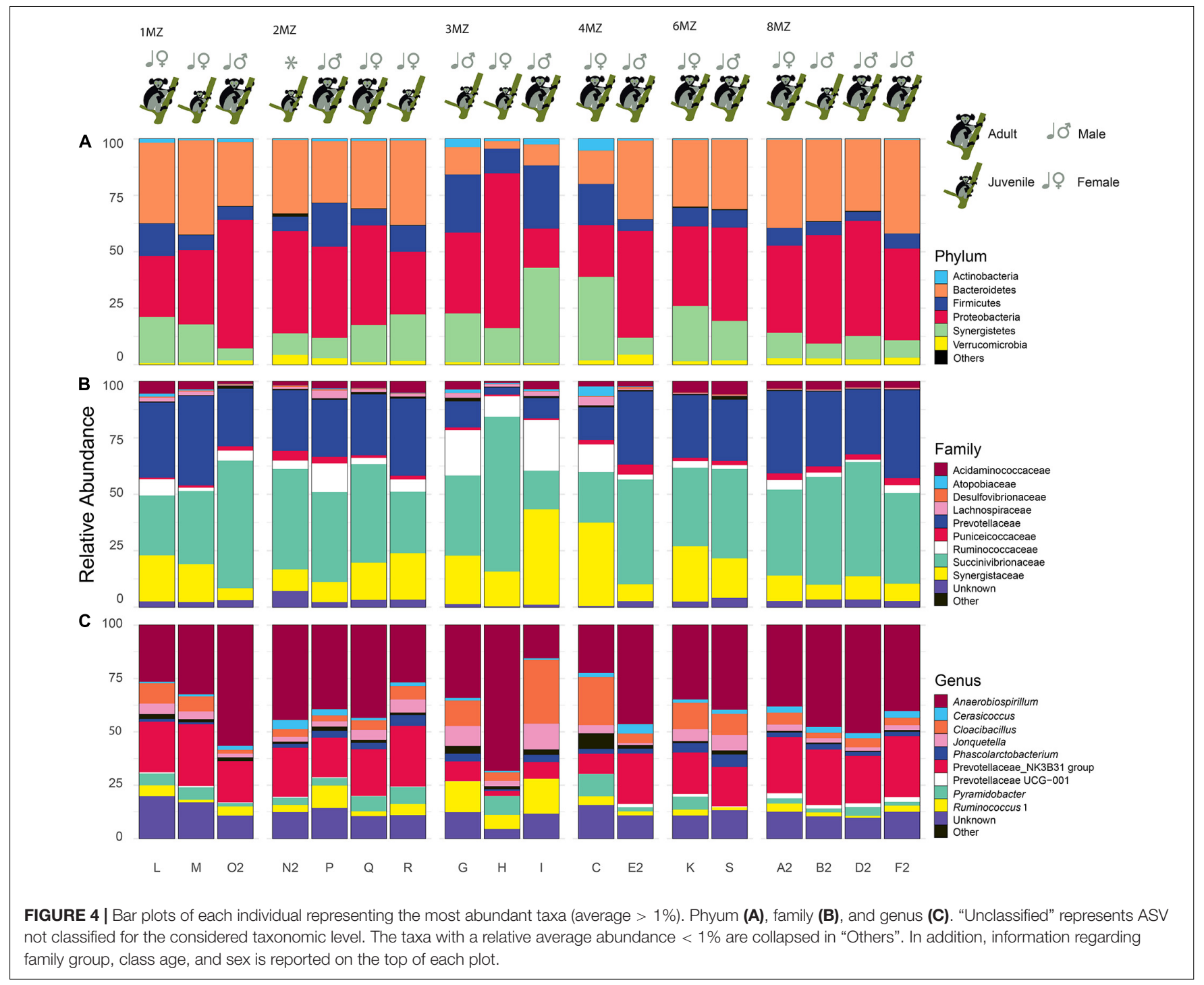

genus Desulfovibrio, characterized the group 2MZ, whereas Actinobacteria with Atopobium and Firmicutes with Tyzzerella 3 were biomarkers of $3 \mathrm{MZ}$ (Figure 6A). Further, Bacteroidetes with Prevotellaceae UCG001 and Verrucomicrobia with Cerasicoccus were more abundant in the group $8 \mathrm{MZ}$ (Figure 6A).

Concerning sex, four AVSs biomarkers were found for females and two AVSs males (Supplementary Table 6). Moreover, Firmicutes and Synergistetes with the genera Cloacibacillus and Jonquetella were more abundant in females; differently, Verrucomicrobia with the genus Cerasicoccus and Proteobacteria with the genus Anaerobiospirillum were mainly present in males (Figure 6B).

\section{DISCUSSION}

\section{Indris Gut Microbiome Diversity}

Although in different proportions, the most abundant phyla found in indris' gut (i.e., Proteobacteria, Bacteroides, and
Firmicutes) are consistent with those found in other studies involving primates (Aivelo et al., 2016). On the other hand, the relative abundance of Proteobacteria found in our study was almost five times higher than that found in other lemurs species, such as Lemur catta (Umanets et al., 2018), Eulemur rufifrons, and E. rubriventer (Bennett et al., 2016; Table 2). Nevertheless, Greene et al. (2020) investigating wild indris' gut microbiome diversity found a higher abundance of Proteobacteria compared to the other three lemur species (i.e., L. catta, E. rufifrons, and E. rubriventer) (Bennett et al., 2016; Umanets et al., 2018), but still lower than what we found in our work (Table 2). With this regard, the high relative abundance of Proteobacteria present in our samples and found in Greene et al. (2020) could represent the typical composition of the gut microbiome of healthy individuals. Differently, in humans, an increased prevalence of Proteobacteria has been observed as a potential signature of dysbiosis (Illiano et al., 2020). Specifically, altered homeostasis, caused by environmental or host factors, such as a low-fiber diet and acute or chronic inflammation, could be a selection driver 


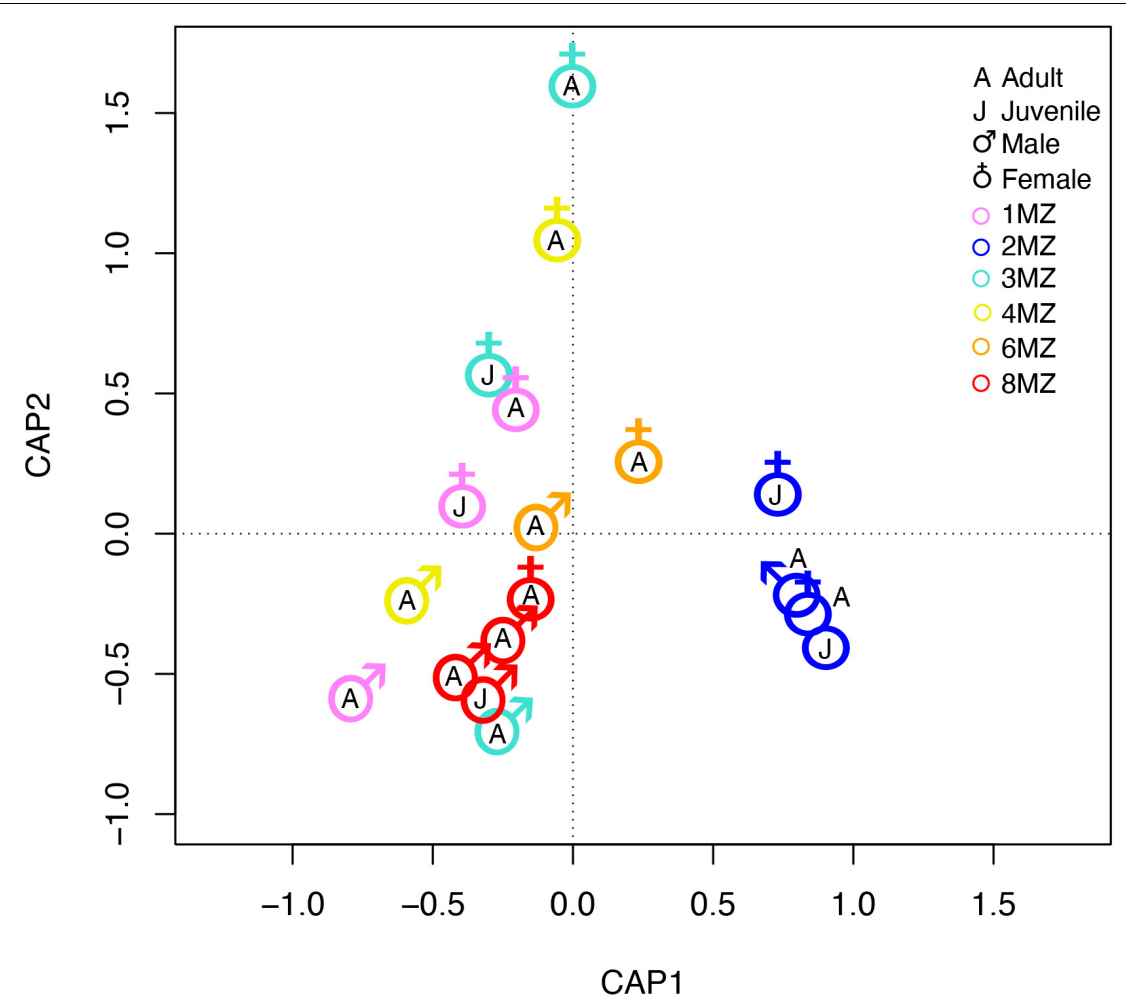

FIGURE 5 | Constrained analysis of principal coordinate ordination plot on bacterial communities of indris fecal samples.

and cause dysbiosis with an increased number of Proteobacteria in the gut. For what concerns the indris, their diet is based on fiber due to its folivores' habitus, with usual consumption of soil as integration. Plant leaves and soil could most likely be an important source of Proteobacteria; in fact, plant leaves, and soil contain about 62 and $36.5 \%$ of Proteobacteria, respectively (Shin et al., 2015). Proteobacteria could play a key role in cinnamates degradation and hydroxycinnamates and hydroxycinnamic acids utilization for energy recovery (Greene et al., 2020). Further, indris might rely primarily on Proteobacteria, and secondly on Bacteroidetes and Firmicutes (e.g., Prevotella and Ruminobacter) for fiber digestion (Biddle et al., 2013). Indeed, Firmicutes members such as Lachnospiraceae and Ruminococcaceae, with some Bacteroidetes, have known fiber fermenting abilities. Interestingly, they have been associated with the production of the appreciated colonocyte nutrient butyrate (Biddle et al., 2013; Meehan and Beiko, 2014). The presence of functionally redundant taxa might support functional stability during ordinary life and possible life disturbance (Vital et al., 2017).

Regarding the factors driving microbial diversity, this study showed the crucial role of social groups in shaping the indris microbiome for the first time. Differences among social groups may be related to feeding and social interactions like grooming, which provide close contact between subjects of the same group (Bennett et al., 2016; Raulo et al., 2018). These mechanisms were identified as relevant factors influencing the microbiome composition of baboons and chimpanzees (Degnan et al., 2012; Tung et al., 2015). A study that analyzed the dynamics of the composition of 10 wild groups in the Maromizaha NAP, comprising the groups sampled in this work, found evidence of only one immigrant female and one immigrant male out of 68 indris over 12 years (Rolle et al., 2021 in press). This very low rate of intergroup mobility limits the number of social partners that indri can have in their lives and, consequently, the intergroup transmission of microorganisms and parasites. In addition, sex was another factor that significantly influenced the microbiome alpha and beta-diversity. Particularly, the higher bacterial Shannon diversity found in females than males could be due to the sex hormones that play a crucial role in sex dimorphism (Haro et al., 2016). Moreover, females showed a higher abundance of Cloacibacillus and Jonquetella, both belonging to the novel phylum Synergistetes, that inhabits the mammalian gastrointestinal tract typically (Jumas-Bilak et al., 2007; Looft et al., 2013). Differently, males had a higher abundance of bacteria from the Anaerobiospirillum genus. This difference can be explained by the fact that females and males differ in nutritional and energetic demands for growth, development, and reproduction. Moreover, sexspecific traits influence the ecological structure of the gut microbiome, maintaining sex differences in physiology and behavior throughout life (Jašarević et al., 2016).

\section{Geophagy in Indris}

Typical Oxisols with a reddish color characterized geophagic and non-geophagic sampling sites. Some inherent characteristics of the Oxisols, such as the quite acidic $\mathrm{pH}$, the richness of 


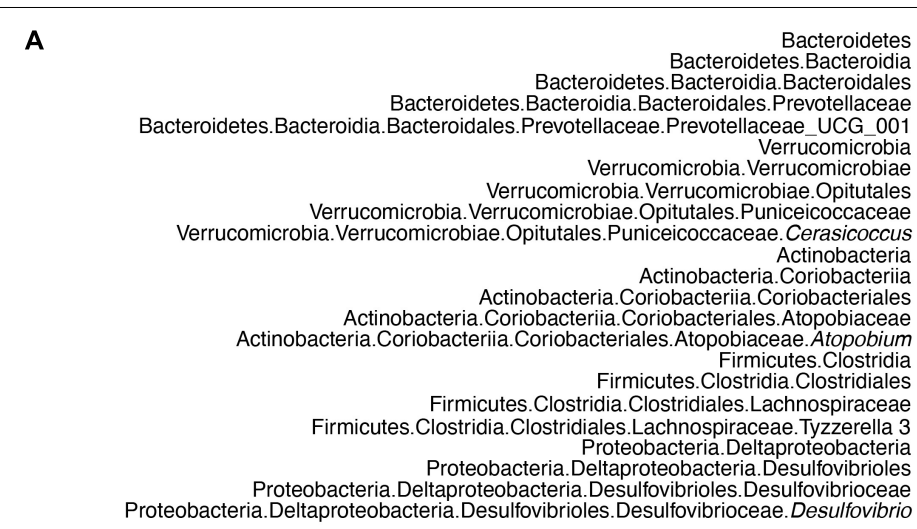

Proteobacteria.Deltaproteobacteria.Desulfovibrioles.Desulfovibrioceae.Desulfovibrio

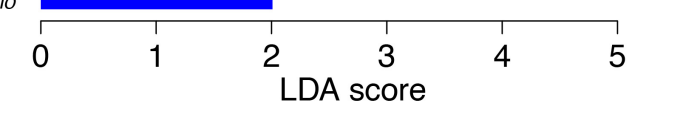

-

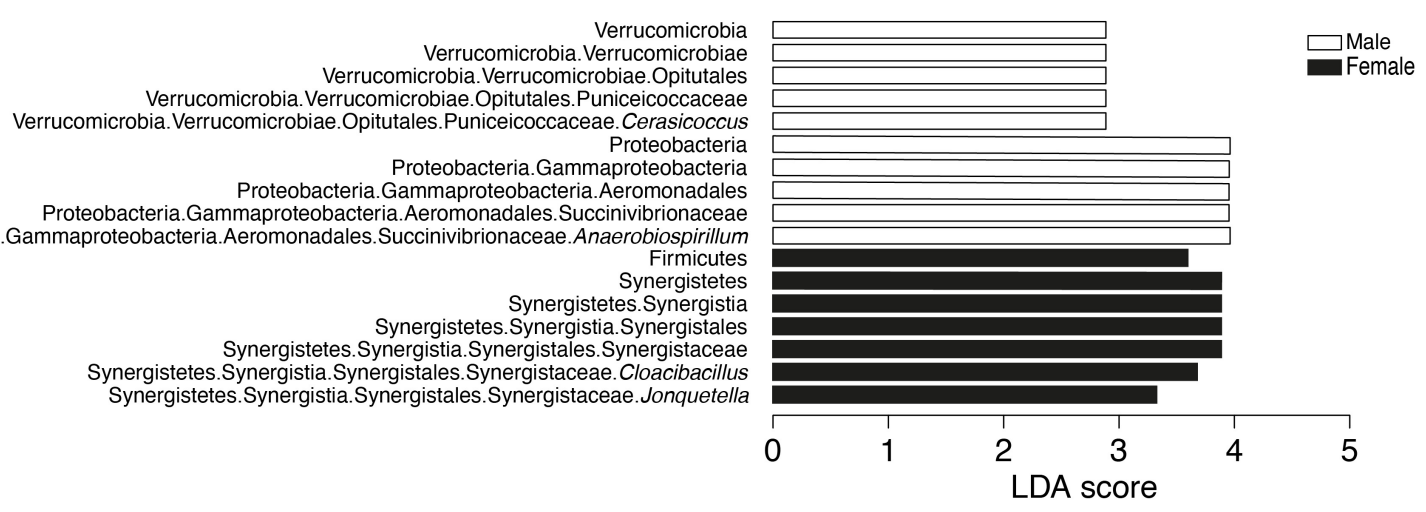

FIGURE 6 | Linear discriminant analysis effect size plots of the biomarkers taxa for the categories family group (A) and sex (B).

TABLE 2 | Percentage of the three top bacterial phyla found in this study and other studies.

\begin{tabular}{|c|c|c|c|c|}
\hline Lemurs species & References & Firmicutes (\%) & Bacteroidetes (\%) & Proteobacteria (\%) \\
\hline Lemur catta & Umanets et al., 2018 & $51.57 \pm 0.11$ & $15.81 \pm 0.11$ & $5.21 \pm 0.11$ \\
\hline Eulemur rufifrons and E. rubriventer & Bennett et al., 2016 & $43.3 \pm 0.064$ & $30.3 \pm 0.053$ & $7.4 \pm 0.031$ \\
\hline I. indri & Greene et al., 2020 & 19.70 & 47.70 & 20.50 \\
\hline I. indri & This study & $11.1 \pm 1.9$ & $28.7 \pm 2.8$ & $40.1 \pm 9.5$ \\
\hline
\end{tabular}

secondary oxide-hydroxides and highly weathered clays, seem more important for geophagy than the content in pseudototal or available elements (Vågen et al., 2006; Borruso et al., 2021). According to the adaptive hypothesis of geophagy, the soil ingested by indri could play a crucial role in micronutrient supplementation and detoxification (i.e., adsorption functions via oxyhydroxides and clays) (Pebsworth et al., 2019). Indeed, indris are folivorous, consuming mainly immature leaves rich in potentially toxic compounds such as tannins, terpenes, and cyanogenic glycosides derived (Hemingway, 1998); thus, the geophagic soil could be involved in the plant's toxin adsorption derived from the diet (de Souza et al., 2002; Pebsworth et al., 2019).

However, the reason behind the selection of one site instead of another one remains unclear. The choice of the sites characterized by the exposition of lower soil horizons could be a strategy to limit the energy expended in obtaining soil from the intact ground.
Nevertheless, some elements (i.e., Ca, S, Na, Cr, B, and available $\mathrm{Ni}$ ) were present at lower concentrations in geophagic than in non-geophagic soil. Although we cannot directly explain these differences, they could indicate that other soil quality traits could orientate the selection of a specific soil.

In conclusion, studies on different species suggested that geophagic sites are required to maintain individual and population health (Pebsworth et al., 2019). Accordingly, preserving the geophagic sites is crucial in wildlife conservation policy.

\section{Microbial Ecology and Indri Conservation}

Microbial ecology offers valuable perspectives to investigate primate health and improve conservation efforts. Understanding the drivers affecting the microbiome associated with the host (e.g., indri) is critical for conservation biology. It is well known that the microbial gut communities profoundly affect host health, 
nutrition, physiology, and immune systems (Sandri et al., 2020). For instance, our study is fundamental to document the typical composition of healthy individuals considering sex and group influence (Amato et al., 2020). Therefore, many studies have been conducted on the human microbiome where microbial biomarkers of health have been shown, such as the presence of Faecalibacterium prausnitzii (Manor et al., 2020). The acquisition of new information about animal gut microbiomes can help identify biomarkers for animal health. In addition, microbial gut communities are sensitive to environmental alterations and their diversity seems to be correlated with habitat quality and, thus, with possible health implications (Scotti et al., 2017). The application of gut microbiome analyses to wildlife conservation of endangered species is currently in its infancy but holds enormous potential. To date, no conservation policy or legislation includes microbiome assessments. Integrating a new understanding of the patterns of microbial diversity and early signs of impending microbial disruption offer valuable tools for informing conservation strategies and monitoring and promoting primate health (Stumpf et al., 2016). The present study represents a first insight toward understanding the overall diversity and ecology of indris microbiome in different familiar groups and a sex-dependent baseline that can be tracked over time as a component of efforts to help animal conservation.

\section{DATA AVAILABILITY STATEMENT}

The datasets presented in this study can be found in online repositories. The names of the repository/repositories and accession number(s) can be found below: Sequence Read Archive (SRA) BioProject ID: PRJNA701813.

\section{ETHICS STATEMENT}

Ethical review and approval was not required for the animal study because. The non-invasive methods used for fecal collections of wild indris adhere to the International Primatological Society (IPS) "Principles for the Ethical Treatment of Non-Human Primates." Field data collection protocols were reviewed and approved by Madagascar's Ministere de l'Environnement, de l'Ecologie et des Forêts (Permit 2018:

\section{REFERENCES}

Aivelo, T., Laakkonen, J., and Jernvall, J. (2016). Population- and individuallevel dynamics of the intestinal microbiota of a small primate. Appl. Environ. Microbiol. 82, 3537-3545. doi: 10.1128/AEM.00559-16

Amaral, W. Z., Lubach, G. R., Proctor, A., Lyte, M., Phillips, G. J., and Coe, C. L. (2017). Social influences on Prevotella and the gut microbiome of young monkeys. Psychosom. Med. 79, 888-897. doi: 10.1097/PSY.0000000000000454

Amato, K. R., Kuthyar, S., Ekanayake-Weber, M., Salmi, R., Snyder-Mackler, N., Wijayathunga, L., et al. (2020). Gut microbiome, diet, and conservation of endangered langurs in Sri Lanka. Biotropica 52, 981-990. doi: 10.1111/btp. 12805

Amato, K. R., Martinez-Mota, R., Righini, N., Raguet-Schofield, M., Corcione, F. P., Marini, E., et al. (2016). Phylogenetic and ecological factors impact the gut microbiota of two Neotropical primate species. Oecologia 180, 717-733. doi: $10.1007 / \mathrm{s} 00442-015-3507-\mathrm{z}$
$\mathrm{N}^{\circ}$ 91/18/MEEF/SG/DGF/DSAP/SCB.Re). Field data collection protocols were also approved by Groupe d'Étude et de Recherche sur les Primates de Madagascar (GERP), the association governing research in the Maromizaha New Protected Area.

\section{AUTHOR CONTRIBUTIONS}

CSa CSp, CG, PM, PT, and LB conceived and designed the experiments. VT and RR supervised the collection of the fecal and soil samples. FC, AC, DL, LC, and MM carried out the experiments. FC, LB, DL, MM, VT, LC, MS, TM, AC, MD, and FB analyzed the data. FC, AC, LB, CSa, CSp, CG, VT, SC, TM, and $\mathrm{PM}$ wrote the manuscript. All authors contributed to the article and approved the submitted version.

\section{FUNDING}

This work was supported by the Open Access Publishing Fund of the Free University of Bozen-Bolzano.

\section{ACKNOWLEDGMENTS}

We are grateful to the Botanist, Rakotozafy M. Andriatsarafara for having contributed to the qualitative study of the soil, Matteo Moretti for the graphics design and Manuela Dasser of Marameolab for graphical illustrations. We thank Chiara De Gregorio and the research guides in Maromizaha (Boto Zafison, Ranaivomanana Jean, and Kotoarisoa Gilbert), for helping in collecting fecal and soil samples and for helping us with the filming. We would also thank Cesare Avesani Zaborra, CEO and Scientific Director of Parco Natura Viva for his continuous support and help to the conservation of Maromizaha Forest fauna and flora.

\section{SUPPLEMENTARY MATERIAL}

The Supplementary Material for this article can be found online at: https://www.frontiersin.org/articles/10.3389/fmicb. 2021.668274/full\#supplementary-material

Bennett, G., Malone, M., Sauther, M. L., Cuozzo, F. P., White, B., Nelson, K. E., et al. (2016). Host age, social group, and habitat type influence the gut microbiota of wild ring-tailed lemurs (Lemur catta). Am. J. Primatol. 78, 883-892. doi: 10.1002/ajp.22555

Biddle, A., Stewart, L., Blanchard, J., and Leschine, S. (2013). Untangling the genetic basis of fibrolytic specialization by Lachnospiraceae and Ruminococcaceae in diverse gut communities. Diversity 5, 627-640. doi: 10.3390/d503 0627

Bocian, C. (1997). Niche separation of Black-and-White Colobus monkeys (Colobus angolensis and C. guereza) in the Ituri Forest. Available online at: https: //www.semanticscholar.org/paper/Niche-separation-of-Black-and-WhiteColobus-monkeys-Bocian/83d202c81a4b27697ab2d6f763edac13b534330d (accessed February 13, 2021).

Bonadonna, G., Torti, V., Gregorio, C. D., Valente, D., Randrianarison, R. M., Pozzi, L., et al. (2019). Evidence of genetic monogamy in the lemur Indri (Indri indri). Am. J. Primatol. 81:e22993. doi: 10.1002/ajp.22993 
Borruso, L., Checcucci, A., Torti, V., Correa, F., Sandri, C., Luise, D., et al. (2021). I like the way you eat it: Lemur (Indri indri) gut mycobiome and geophagy. Microb. Ecol. [Epub ahead of print]. doi: 10.1007/s00248-02001677-5

Britt, A., Randriamandratonirina, N. J., Glasscock, K. D., and Iambana, B. R. (2002). Diet and feeding behaviour of Indri indri in a low-altitude rain forest. Folia Primatol. 73, 225-239. doi: 10.1159/000067455

Callahan, B. J., McMurdie, P. J., Rosen, M. J., Han, A. W., Johnson, A. J. A., and Holmes, S. P. (2016). DADA2: high-resolution sample inference from Illumina amplicon data. Nat. Methods 13, 581-583. doi: 10.1038/nmeth.3869

Campbell, C. J. (2017). "Primates in perspective," in The International Encyclopedia of Primatology. ed. C. J. Campbell (Hoboken, NJ: Wiley).

Cresci, G. A., and Bawden, E. (2015). Gut Microbiome: what we do and don't know. Nutr. Clin. Pract. 30, 734-746. doi: 10.1177/0884533615609899

Davies, A. G., and Baillie, I. C. (1988). Soil-eating by red leaf monkeys (Presbytis rubicunda) in Sabah, Northern Borneo. Biotropica 20, 252-258. doi: 10.2307/ 2388241

De Gregorio, C., Carugati, F., Estienne, V., Valente, D., Raimondi, T., Torti, V., et al. (2021). Born to sing! Song development in a singing primate. Curr. Zool. [Epub ahead of print]. doi: 10.1093/cz/zoab018

De Gregorio, C., Valente, D., Torti, V., Zanoli, A., Colaci Cirillo, D., Bazzano, G., et al. (2019). Song structure and sex specific features in the indris. Proc. Meet. Acoust. 36:010002. doi: 10.1121/2.0001021

de Souza, L. L., Ferrari, S. F., da Costa, M. L., and Kern, D. C. (2002). Geophagy as a correlate of folivory in red-handed howler monkeys (Alouatta belzebul) from eastern Brazilian Amazonia. J. Chem. Ecol. 28, 1613-1621. doi: 10.1023/A: 1019928529879

Degnan, P. H., Pusey, A. E., Lonsdorf, E. V., Goodall, J., Wroblewski, E. E., Wilson, M. L., et al. (2012). Factors associated with the diversification of the gut microbial communities within chimpanzees from Gombe National Park. Proc. Natl. Acad. Sci. U.S.A. 109, 13034-13039. doi: 10.1073/pnas.1110994109

Dew, J. L. (2005). Foraging, food choice, and food processing by sympatric ripefruit specialists: Lagothrix lagotricha poeppigii and Ateles belzebuth belzebuth. Int. J. Primatol. 26, 1107-1135. doi: 10.1007/s10764-005-6461-5

Dixon, P. (2003). VEGAN, a package of R functions for community ecology. J. Veg. Sci. 14, 927-930. doi: 10.1111/j.1654-1103.2003.tb02228.x

Ferrari, S. F., Veiga, L. M., and Urbani, B. (2008). Geophagy in new world monkeys (Platyrrhini): ecological and geographic patterns. Folia Primatol. 79, 402-415. doi: 10.1159/000141901

Fogel, A. T. (2015). The gut microbiome of wild lemurs: a comparison of sympatric Lemur catta and Propithecus verreauxi. Folia Primatol. 86, 85-95. doi: 10.1159/ 000369971

Gamba, M., Torti, V., Estienne, V., Randrianarison, R. M., Valente, D., Rovara, P., et al. (2016). The Indris have got rhythm! timing and pitch variation of a primate song examined between sexes and age classes. Front. Neurosci. 10:249. doi: $10.3389 /$ fnins.2016.00249

Geilfus, C.-M. (2019). "Plant secondary compounds," in Controlled Environment Horticulture: Improving Quality of Vegetables and Medicinal Plants, ed. C.-M. Geilfus (Cham: Springer International Publishing), 19-33. doi: 10.1007/978-3030-23197-2_3

Greene, L. K., Williams, C. V., Junge, R. E., Mahefarisoa, K. L., Rajaonarivelo, T., Rakotondrainibe, H., et al. (2020). A role for gut microbiota in host niche differentiation. ISME J. 14, 1675-1687. doi: 10.1038/s41396-0200640-4

Grieneisen, L. E., Charpentier, M. J. E., Alberts, S. C., Blekhman, R., Bradburd, G., Tung, J., et al. (2019). Genes, geology and germs: gut microbiota across a primate hybrid zone are explained by site soil properties, not host species. Proc. R. Soc. B Biol. Sci. 286:20190431. doi: 10.1098/rspb.2019. 0431

Hale, V. L., Tan, C. L., Niu, K., Yang, Y., Knight, R., Zhang, Q., et al. (2018). Diet versus phylogeny: a comparison of gut microbiota in captive colobine monkey species. Microb. Ecol. 75, 515-527. doi: 10.1007/s00248-0171041-8

Hale, V. L., Tan, C. L., Niu, K., Yang, Y., Zhang, Q., Knight, R., et al. (2019). Gut microbiota in wild and captive Guizhou snub-nosed monkeys, Rhinopithecus brelichi. Am. J. Primatol. 81:e22989. doi: 10.1002/ajp.22989

Haro, C., Rangel-Zúñiga, O. A., Alcalá-Díaz, J. F., Gómez-Delgado, F., PérezMartínez, P., Delgado-Lista, J., et al. (2016). Intestinal microbiota is influenced by gender and body mass index. PLoS One 11:e0154090. doi: 10.1371/journal. pone. 0154090

Heinen, J., and Mehta, J. (1999). Conceptual and legal issues in the designation and management of conservation areas in Nepal. Environ. Conserv. 26, 21-29. doi: 10.1017/S0376892999000053

Hemingway, C. A. (1998). Selectivity and variability in the diet of Milne-Edwards' sifakas (Propithecus diadema edwardsi): implications for folivory and seedeating. Int. J. Primatol. 19, 355-377. doi: 10.1023/A:1020344018670

Huffman, M. A., Gotoh, S., Turner, L. A., Hamai, M., and Yoshida, K. (1997). Seasonal trends in intestinal nematode infection and medicinal plant use among chimpanzees in the Mahale Mountains, Tanzania. Primates 38, 111-125. doi: 10.1007/BF02382002

Illiano, P., Brambilla, R., and Parolini, C. (2020). The mutual interplay of gut microbiota, diet and human disease. FEBS J. 287, 833-855. doi: 10.1111/febs. 15217

Jašarević, E., Morrison, K. E., and Bale, T. L. (2016). Sex differences in the gut microbiome-brain axis across the lifespan. Philos. Trans. R. Soc. B. Biol. Sci. 371:20150122. doi: 10.1098/rstb.2015.0122

Jumas-Bilak, E., Carlier, J.-P., Jean-Pierre, H., Citron, D., Bernard, K., Damay, A., et al. (2007). Jonquetella anthropi gen. nov., sp. nov., the first member of the candidate phylum "Synergistetes" isolated from man. Int. J. Syst. Evol. Microbiol. 57, 2743-2748. doi: 10.1099/ijs.0.65213-0

King, T., Dolch, R., Randriahaingo, H. N. T., Randrianarimanana, L., and Ravaloharimanitra, M. (2020). IUCN Red List of Threatened Species: Indri indri. IUCN Red List Threat. Species. Available online at: https://dx.doi.org/10.2305/ IUCN.UK.2020-2.RLTS.T10826A115565566.en (accessed June 19, 2021).

Krishnamani, R., and Mahaney, W. C. (2000). Geophagy among primates: adaptive significance and ecological consequences. Anim. Behav. 59, 899-915. doi: 10. 1006/anbe.1999.1376

Lankau, E. W., Hong, P.-Y., and Mackie, R. I. (2012). Ecological drift and local exposures drive enteric bacterial community differences within species of Galápagos iguanas. Mol. Ecol. 21, 1779-1788. doi: 10.1111/j.1365-294X.2012. 05502.x

Li, H., Li, T., Yao, M., Li, J., Zhang, S., Wirth, S., et al. (2016). Pika gut may select for rare but diverse environmental bacteria. Front. Microbiol. 7:1269. doi: $10.3389 /$ fmicb.2016.01269

Lombardo, M. P. (2008). Access to mutualistic endosymbiotic microbes: an underappreciated benefit of group living. Behav. Ecol. Sociobiol. 62, 479-497. doi: 10.1007/s00265-007-0428-9

Looft, T., Levine, U. Y., and Stanton, T. B. (2013). Cloacibacillus porcorum sp. nov., a mucin-degrading bacterium from the swine intestinal tract and emended description of the genus Cloacibacillus. Int. J. Syst. Evol. Microbiol. 63, 19601966. doi: 10.1099/ijs.0.044719-0

Manor, O., Dai, C. L., Kornilov, S. A., Smith, B., Price, N. D., Lovejoy, J. C., et al. (2020). Health and disease markers correlate with gut microbiome composition across thousands of people. Nat. Commun. 11:5206. doi: 10.1038/s41467-02018871-1

McMurdie, P. J., and Holmes, S. (2013). phyloseq: an R package for reproducible interactive analysis and graphics of microbiome census data. PLoS One 8:e61217. doi: 10.1371/journal.pone.0061217

Meehan, C. J., and Beiko, R. G. (2014). A phylogenomic view of ecological specialization in the Lachnospiraceae, a family of digestive tract-associated bacteria. Genome Biol. Evol. 6, 703-713. doi: 10.1093/gbe/evu050

Moeller, A. H., Peeters, M., Ndjango, J.-B., Li, Y., Hahn, B. H., and Ochman, H. (2013). Sympatric chimpanzees and gorillas harbor convergent gut microbial communities. Genome Res. 23, 1715-1720. doi: 10.1101/gr.154773.113

Norconk, M. A., Wright, B. W., Conklin-Brittain, N. L., and Vinyard, C. J. (2009). "Mechanical and nutritional properties of food as factors in platyrrhine dietary adaptations," in South American Primates: Comparative Perspectives in the Study of Behavior, Ecology, and Conservation Developments in Primatology: Progress and Prospects, eds P. A. Garber, A. Estrada, J. C. Bicca-Marques, E. W. Heymann, and K. B. Strier (New York, NY: Springer), 279-319. doi: 10.1007/ 978-0-387-78705-3_11

Overdorff, D. J. (1993). Similarities, differences, and seasonal patterns in the diets of Eulemur rubriventer and Eulemur fulvus rufus in the Ranomafana National Park, Madagascar. Int. J. Primatol. 14, 721-753. doi: 10.1007/BF02192188

Pebsworth, P. A., Huffman, M. A., Lambert, J. E., and Young, S. L. (2019). Geophagy among nonhuman primates: a systematic review of current 
knowledge and suggestions for future directions. Am. J. Phys. Anthropol. 168, 164-194. doi: 10.1002/ajpa.23724

Perofsky, A. C., Lewis, R. J., and Meyers, L. A. (2019). Terrestriality and bacterial transfer: a comparative study of gut microbiomes in sympatric Malagasy mammals. ISME J. 13, 50-63. doi: 10.1038/s41396-018-0251-5

Pollock, J. I. (1979). Female dominance in Indri indri. Folia Primatol. 31, 143-164. doi: $10.1159 / 000155877$

Powzyk, J. A. (1997). The Socio-Ecology Of Two Sympatric Indriids: Propithecus Diadema Diadema And Indri indri, A Comparison Of Feeding Strategies And Their Possible Repercussions On Species-Specific Behaviors. Ph.D. thesis. Durham: Duke University.

Powzyk, J. A., and Mowry, C. B. (2003). Dietary and feeding differences between sympatric Propithecus diadema diadema and Indri indri. Int. J. Primatol. 24, 1143-1162. doi: 10.1023/B:IJOP.0000005984.36518.94

Quast, C., Pruesse, E., Yilmaz, P., Gerken, J., Schweer, T., Yarza, P., et al. (2013). The SILVA ribosomal RNA gene database project: improved data processing and web-based tools. Nucleic Acids Res. 41, D590-D596. doi: 10.1093/nar/gks1219

R Core Team (2021). R: A Language And Environment For Statistical Computing.Vienna, VIE: R Foundation for Statistical Computing.

Raulo, A., Ruokolainen, L., Lane, A., Amato, K., Knight, R., Leigh, S., et al. (2018). Social behaviour and gut microbiota in red-bellied lemurs (Eulemur rubriventer): in search of the role of immunity in the evolution of sociality. J. Anim. Ecol. 87, 388-399. doi: 10.1111/1365-2656.12781

Rolle, F., Torti, V., Valente, D., De Gregorio, C., Giacoma, C., and von Hardenberg, A. (2021). Sex and age-specific survival and life expectancy in a free ranging population of Indri indri (Gmelin, 1788). Eur. Zool. J. [Epub ahead of print]. doi: $10.1080 / 24750263.2021 .1947398$

Sandri, C., Correa, F., Spiezio, C., Trevisi, P., Luise, D., Modesto, M., et al. (2020). Fecal microbiota characterization of seychelles giant tortoises (Aldabrachelys gigantea) living in both wild and controlled environments. Front. Microbiol. 11:569249. doi: $10.3389 /$ fmicb.2020.569249

Scotti, E., Boué, S., Sasso, G. L., Zanetti, F., Belcastro, V., Poussin, C., et al. (2017). Exploring the microbiome in health and disease: implications for toxicology. Toxicol. Res. Appl. 1:2397847317741884. doi: 10.1177/2397847317741884

Segata, N., Izard, J., Waldron, L., Gevers, D., Miropolsky, L., Garrett, W. S., et al. (2011). Metagenomic biomarker discovery and explanation. Genome Biol. 12:R60. doi: 10.1186/gb-2011-12-6-r60

Shin, N.-R., Whon, T. W., and Bae, J.-W. (2015). Proteobacteria: microbial signature of dysbiosis in gut microbiota. Trends Biotechnol. 33, 496-503. doi: 10.1016/j.tibtech.2015.06.011

Shreiner, A. B., Kao, J. Y., and Young, V. B. (2015). The gut microbiome in health and in disease. Curr. Opin. Gastroenterol. 31, 69-75. doi: 10.1097/MOG. 0000000000000139

Sparks, D. L., Page, A. L., Helmke, P. A., Loeppert, R. H., Soltanpour, P. N., Tabatabai, M. A., et al. (1996). Methods of Soil Analysis: Part 3. Madison, MDN: Soil Science Society of America.

Stumpf, R. M., Gomez, A., Amato, K. R., Yeoman, C. J., Polk, J. D., Wilson, B. A., et al. (2016). Microbiomes, metagenomics, and primate conservation: new strategies, tools, and applications. Biol. Conserv. 199, 56-66. doi: 10.1016/ j.biocon.2016.03.035

Takahashi, S., Tomita, J., Nishioka, K., Hisada, T., and Nishijima, M. (2014). Development of a prokaryotic universal primer for simultaneous analysis of bacteria and archaea using next-generation sequencing. PLoS One 9:e105592. doi: 10.1371/journal.pone.0105592

Torti, V., Miaretsoa, L., Valente, D., De Gregorio, C., Bonadonna, C., Randrianarison, R. M., (2019). "Indri indri (Gmelin, 1788)," in Primates in Peril: The World's 25 Most Endangered Primates 2018-2020, eds C. Schwitzer, R. A. Mittermeier, A. B. Rylands, F. Chiozza, E. A. Williamson, D. Byler, S. Wich, T. Humle, C. Johnson, H. Mynott, and G. McCabe (Washington, DC: IUCN SSC Primate Specialist Group, International Primatological Society, Global Wildlife Conservation, and Bristol Zoological Society), 24-27.

Torti, V., Gamba, M., Rabemananjara, Z. H., and Giacoma, C. (2013). The songs of the indris (Mammalia: Primates: Indridae): contextual variation in the longdistance calls of a lemur. Ital. J. Zool. 80, 596-607. doi: 10.1080/11250003.2013. 845261

Tung, J., Barreiro, L. B., Burns, M. B., Grenier, J.-C., Lynch, J., Grieneisen, L. E., et al. (2015). Social networks predict gut microbiome composition in wild baboons. eLife 4:e05224. doi: 10.7554/eLife.05224

Umanets, A., de Winter, I., IJdema, F., Ramiro-Garcia, J., van Hooft, P., Heitkönig, I. M. A., et al. (2018). Occupancy strongly influences faecal microbial composition of wild lemurs. FEMS Microbiol. Ecol. 94:fiy017. doi: 10.1093/ femsec/fiy017

Vågen, T.-G., Shepherd, K. D., and Walsh, M. G. (2006). Sensing landscape level change in soil fertility following deforestation and conversion in the highlands of Madagascar using Vis-NIR spectroscopy. Geoderma 133, 281-294. doi: 10. 1016/j.geoderma.2005.07.014

Vital, M., Karch, A., and Pieper, D. H. (2017). Colonic butyrate-producing communities in humans: an overview using omics data. mSystems 2:e00130-17. doi: 10.1128/mSystems.00130-17

Young, S. L., Sherman, P. W., Lucks, J. B., and Pelto, G. H. (2011). Why on earth?: evaluating hypotheses about the physiological functions of human geophagy. Q. Rev. Biol. 86, 97-120. doi: 10.1086/659884

Zhou, D., Bai, Z., Zhang, H., Li, N., Bai, Z., Cheng, F., et al. (2018). Soil is a key factor influencing gut microbiota and its effect is comparable to that exerted by diet for mice. F1000Research 7:1588. doi: 10.12688/f1000research.15297.1

Conflict of Interest: The authors declare that the research was conducted in the absence of any commercial or financial relationships that could be construed as a potential conflict of interest.

Publisher's Note: All claims expressed in this article are solely those of the authors and do not necessarily represent those of their affiliated organizations, or those of the publisher, the editors and the reviewers. Any product that may be evaluated in this article, or claim that may be made by its manufacturer, is not guaranteed or endorsed by the publisher.

Copyright (C) 2021 Correa, Torti, Spiezio, Checcucci, Modesto, Borruso, Cavani, Mimmo, Cesco, Luise, Randrianarison, Gamba, Rarojoson, Sanguinetti, Di Vito, Bugli, Mattarelli, Trevisi, Giacoma and Sandri. This is an open-access article distributed under the terms of the Creative Commons Attribution License (CC BY). The use, distribution or reproduction in other forums is permitted, provided the original author(s) and the copyright owner(s) are credited and that the original publication in this journal is cited, in accordance with accepted academic practice. No use, distribution or reproduction is permitted which does not comply with these terms. 